\title{
Paper-based synthesis of Prussian Blue Nanoparticles for the development of whole blood glucose electrochemical biosensor ${ }^{\text {tw }}$
}

\author{
Stefano Cinti*, Roberto Cusenza, Danila Moscone, Fabiana Arduini \\ Department of Chemical Science and Technologies, University of Rome "Tor Vergata", Via della Ricerca Scientifica 1, 00133 Rome, Italy
}

\section{A R T I C L E I N F O}

\section{Keywords:}

Biosensor

Paper-based

Prussian Blue Nanoparticles

Screen-printed electrodes

Point-of-care

Whole blood

\begin{abstract}
A B S T R A C T
Nowadays, environmentally friendly synthesis pathways for preserving the environment and minimizing waste are strongly required. Herein, we propose filter paper as a convenient scaffold for chemical reactions. To demonstrate this novel approach, Prussian Blue Nanoparticles (PBNPs) were synthesized on filter paper by utilizing few $\mu \mathrm{L}$ of its precursors without external inputs, i.e. $\mathrm{pH}$, voltage, reducing agents, and without producing waste as well. The functional paper, named "Paper Blue", is successfully applied in the sensing field, exploiting the reduction of hydrogen peroxide at low applied potential. The eco-designed "Paper Blue" was combined with wax- and screen-printing to manufacture a reagentless electrochemical point-of-care device for diabetes selfmonitoring, by using glucose oxidase as the biological recognition element. Blood glucose was linearly detected for a wide concentration range up to $25 \mathrm{mM}(450 \mathrm{mg} / \mathrm{dL})$, demonstrating its suitability for management of diabetes and glucose-related diseases. The Paper Blue-based biosensor demonstrated a correlation coefficient of 0.987 with commercial glucose strips (Bayer Contour XT). The achieved results demonstrated the effectiveness of this approach, which is also extendible to other (bio)systems to be applied in catalysis, remediation, and diagnostics.
\end{abstract}

\section{Introduction}

Paper is establishing itself as one of the most intriguing materials of the 21th century, being adopted in various fields, as paper is used in applications such as building batteries, amplifying nucleic acids, remediating pollution, and fabricating low-cost diagnostics, etc [1-4]. Among its valuable features, paper is inexpensive, lightweight, abundant, locally producible, biodegradable, and recyclable $[5,6]$. These features make paper an ideal sustainable material since i) environmentally, paper minimizes waste production, and can be easily burned, ii) economically, replacing plastic with paper lowers production costs (at laboratory scale) by 50\% [7], and iii) socially [8] paper can improve quality of life and safety, i.e. portable diagnostics.

Although both filter, copy, and glossy papers satisfy the above listed features, filter paper is the majorly exploited one because of its easily funtionalizable 3D cellulosic network. The functionalized paper can be tailored depending on research interests, i.e. energy, printed electronics, remediation, sensing. Even if various paper-based analytical devices have been reported in literature, an interesting approach involving the use of paper has been reported in 2012 by $\mathrm{Li}$ and colleagues [9]. Inspired from Rowling's novel "Harry Potter and the Chamber of
Secrets", they took advantage of the paper porosity to develop an "in writing" paper-based tool for blood group analysis exploiting specific reactions.

Even though paper has brought fantasy to the scientific community, its role as scaffold for chemical reactions has not been reported yet. According to some of the 12 Principles of Green Chemistry established by Anastas and Warner [10] paper owns numerous green-features, highlighting itself as a sustainable reactor: the use of tiny amount of precursors prevents waste (1st), solvents are unnecessary (5th), being equipment-free, means that no energy is required (6th), and it allows for real-time in process monitoring (11th). Herein, we used filter paper to synthesize Prussian Blue Nanoparticles (PBNPs) as a case of study. With respect to the state of the art, filter paper does not require external stimuli as pH adjustment, applied voltage or current, ultrasound, reducing agents [11-13]. The morphological investigation has revealed the formation of homogenous nanoparticles onto filter paper, producing what we called "Paper Blue". In combination with printing technologies, "Paper Blue" was used to develop a new-class of printed diagnostic tool for diabetes management. Whole blood glucose is reagentlessly monitored with performances comparable to those of commercially available glucose strips.

\footnotetext{
This paper is dedicated to Professor Giuseppe Palleschi on the occasion of his 70th birthday.

* Corresponding author.

E-mail address: Stefano.cinti@uniroma2.it (S. Cinti).
} 
Prussian Blue, also known as ferric hexacyanoferrate, is a very effective material in catalyzing the reduction of hydrogen peroxide [14] and it is frequently involved in the biosensors development to evaluate the level of glucose. Briefly, Prussian Blue is used to realize glucose oxidase-based biosensors since it is capable to detect hydrogen peroxide at low applied potential with high sensitivity and selectivity, which is by-produced from the oxidation of glucose by glucose oxidase. Herein, for the first time the porous structure of filter paper is exploited both to synthesize the electrocatalyst, i.e. PBNPs, and to load all the reagents necessary to perform the analytical assay $[15,16]$, i.e. buffer, electrolyte, glucose oxidase. However, the combination of Prussian Blue with glucose oxidase has been recently investigated by other research groups. In 2017, Valiūnienè et al. used Prussian Blue together with polypyrrole to entrap glucose oxidase for the measurement of glucose [17]. In their study, authors investigated the properties of the hybrid layer (Prussian Blue, polypyrrole, glucose oxidase) during the detection of glucose. Thanks to the electrochemical Fast Fourier transformation (FFT)-based impedance spectroscopy, they observed how the properties of the hybrid layer drop casted onto a graphite electrode did not reveal significant changes in presence of different amount of glucose. Their findings could be relevant for selecting the proper strategy for developing novel glucose biosensors with a lowered inactivation rate of the platforms. The same hybrid composite has been used to realize a glucose biosensor using metallurgical titanium as the substrate [18]. Authors evaluated several strategies to form the bioselective layer on titanium electrode. The deposition of Prussian Blue was approached spontaneously or electrochemically, while glucose oxidase was immobilized by electropolymerizing polypyrrole or by cross-linking within vapors of glutaraldehyde. They reported a high linearity for amperometric glucose detection up to $68 \mathrm{mM}$, except when Prussian Blue was electrochemically deposited and glucose oxidase entrapped in polypyrrole (linearity up to $52 \mathrm{mM}$ ). These biosensors required the addition of buffer and the supporting electrolyte to measure glucose (in standard solution). Paper Blue, instead, goes beyond the state of the art since all the required reagents have been loaded within the paper prior to perform the detection.

However, the use of Prussian Blue is not only related to the detection of glucose. Its high sensitivity and specificity towards hydrogen peroxide detection is well-established in literature, and it can be applied to diverse oxidase enzymes, i.e. cholesterol, alcohol, lactate. [19-21].

In this study, we present paper as a reactor for nanoparticle synthesis. To evidence this role, the synthesis of PBNPs has been taken into account. Generally, to form Prussian Blue, a mixture of ferricyanide/Fe(II) salt or ferrocyanide/Fe(III) salts should react together. One may even use just ferri- or ferrocyanide salt, but an external output will be required, i.e. acidic conditions, reductant, voltage, ultrasound [11-13]. Filter paper allows the precursors to get in contact in a favourable micro-environment. Due to the cellulosic fibers and their impregnability, they offer multiple anchor points for nanoparticle formation. This mechanism is autonomous and purely ascribable to the paper's structure. Paper Blue, combined with wax- and screen-printing, served as the substrate for the development of electrochemical reagentfree point-of-care device for diabetes self-monitoring. It demonstrated a very satisfactory correlation coefficient, equal to 0.987 , with commercial glucose strips (Bayer Contour XT).

\section{Experimental}

\subsection{Chemicals and Instruments}

Glucose, glucose oxidase (GOx, from Aspergillus niger), potassium ferricyanide $\left(\mathrm{K}_{3} \mathrm{Fe}(\mathrm{CN})_{6}\right)$, iron chloride $\left(\mathrm{FeCl}_{3}\right)$, potassium chloride, phosphate buffered solution, hydrogen peroxide, ascorbic acid, acetaminophen, and uric acid, were purchased from Sigma-Aldrich (St. Louis, MO). All reagents were used without further purification. Whole blood samples were provided from volunteers. Electrochemical measurements were carried out using a portable EmStat ${ }^{3}$ Instrument (PalmSens, The Netherlands) connected to a laptop. The morphology was investigated by observation with field emission scanning electron microscopy (FEG-SEM, Leo Supra 35, Cambridge, UK).

\subsection{Wax printing}

Prior to obtain Paper Blue, filter paper $\left(67 \mathrm{~g} / \mathrm{m}^{2}\right.$, Cordenons, Italy) is patterned with a solid-ink printer (Xerox ColorQube 8580). A hydrophilic area is surrounded by wax (hydrophobic). Waxed paper is cured for 2' at $100^{\circ} \mathrm{C}$ in a oven to let the wax melts into the paper, creating a 3D-wax barrier.

\subsection{Paper Blue synthesis}

The hydrophilic area is impregnated with the precursors' mixture (10 $\mu \mathrm{L}$ containing $0.05 \mathrm{M} \mathrm{K}_{3} \mathrm{Fe}(\mathrm{CN})_{6}$ and $0.05 \mathrm{M} \mathrm{FeCl}_{3}$, prepared in water). $1.5 \mathrm{~h}$ is enough to synthesize PBNPs on paper, namely Paper Blue.

\subsection{Screen-printing}

A three-electrode configuration is manually screen-printed using a squeegee. $\mathrm{Ag} / \mathrm{AgCl}$ ink (Electrodag $477 \mathrm{SS}$, Acheson, Italy) is used to print the connections and the reference electrode, and successively, the working and counter electrodes are obtained by screen-printing carbon ink (Electrodag 421, Acheson, Italy). Each ink requires to be cured in the oven at $50^{\circ} \mathrm{C}$ for $20^{\prime}$.

\section{Results and discussion}

In this study, to produce nanoparticles, i.e. PBNPs, the filter paper is impregnated with just $10 \mu \mathrm{L}$ of a precursor's mixture $(0.05 \mathrm{M}$ potassium ferricyanide/ferric chloride) prepared in water, Fig. 1.

To demonstrate the active role of the paper, we drop casted the precursors onto a plastic sheet and no change was observed. This could be because both the precursors are in the ferric state, i.e. potassium ferricyanide and ferric chloride, and PB cannot be formed. In fact, PB is typically formed by the electrochemical reduction of a solution that contains both ferric species or by mixing Fe(III) (or Fe(II)) and hexacyanoferrate(II) (or hexacyanoferrate(III)) ions. However, PB is formed when the reaction is carried out within the structure of filter paper, without applying a reducing potential or by mixing different

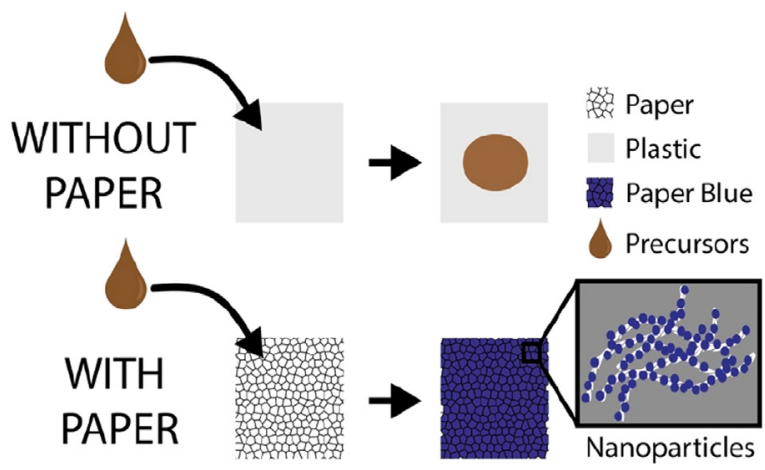

Fig. 1. Role of paper as reactor for Nanoparticles synthesis. The difference between plastic-based and paper-based substrates towards Prussian Blue Nanoparticles synthesis is represented. In the upper part of the figure, it is clear that the use of a plastic-based substrate (grey spot), where the precursors (brown droplet) are drop casted, does not produce Prussian Blue. On the other hand, the use of filter paper (porous white spot), as the scaffold, leads to an obvious formation of homogeneous Prussian Blue Nanoparticles (displayed as the Blue spot) all around the cellulosic fibers (showed in the lower inset). 

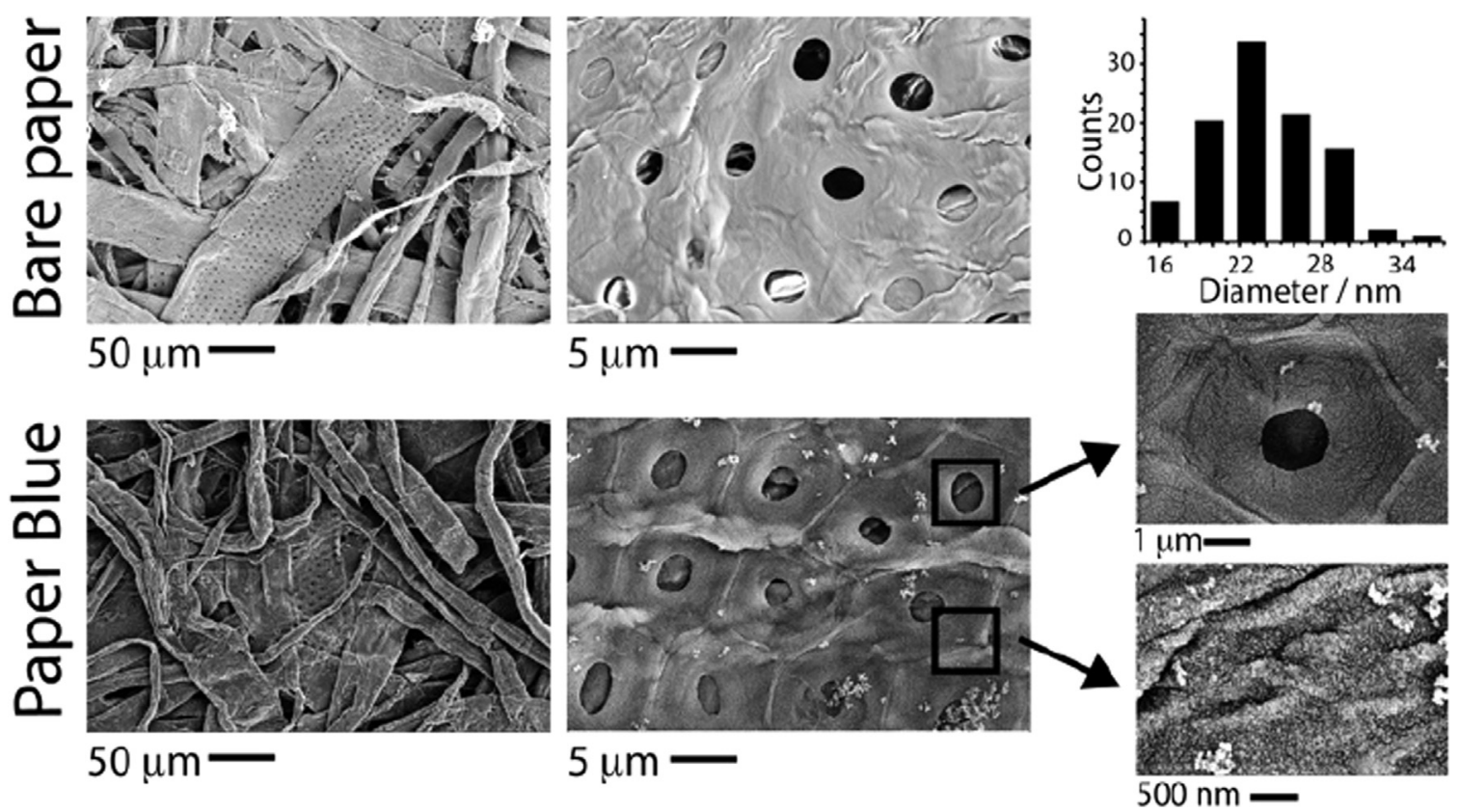

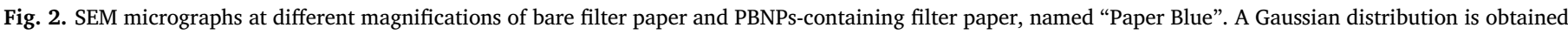
by counting NPs with the ImageJ software.

valence state iron atoms. This behavior is ascribable to the nature of the substrate that hosts the precursors; we assume that the presence of reducing agents in mineral fillers allows for an auto-reduction, similar to those observed when using graphite or carbon black as a support $[14,22]$.

The formation of PBNPs turns the common filter paper into "Paper Blue", highlighted by scanning electron microscopy and displayed in Fig. 2.

The micrographs reveal a homogeneous coverage of the filter paper by these nanoparticles. It is interesting to observe how filter paper results to be composed by rounded holes, which are surrounded by numerous nanosized objects. The mean size of these nano-objects has been evaluated with ImageJ software. Experimentally, as shown in Fig. 2, the maximum of the Gaussian distribution is centered on $25 \mathrm{~nm}$ with a standard deviation of $4 \mathrm{~nm}$. By observing the micrographs reported in Fig. 2, the presence of bigger aggregates is also visible. However, these aggregates are quite scattered and do not meaningfully influence the PBNPs distribution.

Highlighting the role of filter paper as a sustainable reactor for NPs synthesis, its homogenous functionalization leads to several applications. The usefulness of Paper Blue can be easily demonstrated with applications in the diagnostic field, since PB is largely utilized in the development of biosensing tools. Specifically, due to its high selectivity toward the detection of hydrogen peroxide at low overpotential (close to $0 \mathrm{~V}$ ), $\mathrm{PB}$ is often used in combination with the glucose oxidase enzyme because this enzyme catalyzes the oxidation with the production of gluconolactone and hydrogen peroxide as by-products. The monitoring of glucose is fundamental for people suffering from diabetes and related diseases. Glucose oxidase is often adopted as the bio-element in the strips for diabetes self-monitoring [23]. Moreover, considering that $>400$ million people around the world suffer from this illness, this glucose self-test accounts for nearly $85 \%$ of the entire biosensors market. With the market value surpassing \$12 billion [24], Paper Blue can represent a substantial input for the diabetes healthcare challenge in a sustainable manner.

Paper Blue serves as the substrate to develop sustainable glucose strips, combining printed technologies: wax- and screen-printing. The former is a green approach to define the testing area [25], the latter is used to print the sensing strips on the wax-patterned support [26], as shown in Fig. 3.

In details, wax-based inks offer a green tool for isolating the hydrophilic area where PBNPs will be autonomously synthesized. On this area, electrodes are then screen-printed. Paper Blue represents a new concept for analytical tools development: our idea is to provide a set of smart functionalized papers that not only allow to manufacture colorimetric and electrochemical devices, but also avoid ink treatments, i.e. sensing element/s integration, oversetting the classic existing approach in electrochemical sensors fabrication (electrodes are printed and then modified)

To be applied for diabetes healthcare, Paper Blue has to demonstrate its sensitivity towards hydrogen peroxide: its determination indirectly quantifies glucose level. Once screen-printed, Paper Blue's analytical performances are evaluated in presence of increasing concentration of hydrogen peroxide as reported in Fig. 4.

Fig. 4A highlights the typical behavior of PB. The dashed line is acquired in buffer solution and reveals the reduction and oxidation peaks, i.e. i) oxidation of Prussian White (PW) to PB, ii) oxidation of $\mathrm{PB}$ to Berlin Green (BG), iii) reduction of $B G$ to $P B$, iv) reduction of $P B$ to $\mathrm{PW}$. In particular, the oxidized form and the reduced form of $\mathrm{PB}$ are capable to electrocatalytically oxidize and reduce hydrogen peroxide, respectively, as shown in Fig. 4A: intensity of peaks ii) and iv) increases. PB-PW couple is chosen to detect hydrogen peroxide. In Fig. 4B, the increase of hydrogen peroxide level leads to an increase of the cathodic current due to the PW-catalyzed reduction. Paper Blue-based sensor gives a linear response up to $5 \mathrm{mM}$, with a sensitivity of $1.70 \mu \mathrm{A} / \mathrm{mM}$, and a calculated detection limit of $0.17 \mathrm{mM}$. To develop reagent-free point-of-care device for the self-management of diabetes, the porosity of the paper was exploited to store the reagents, as shown in Fig. 5A.

For this reason, the Paper Blue-based sensor is pre-loaded with a glucose oxidase solution prepared in buffered solution, i.e. $5 \mu \mathrm{L}$ of $0.1 \mathrm{mg} / \mathrm{mL}$. It means the end-user will not need to add any chemicals to carry out the test, because all the reagents are already present within the filter paper. After the Paper Blue-based biosensor is dried, $10 \mu \mathrm{L}$ of the sample are added and analyzed. As shown in Fig. 5B, the increase of glucose concentration leads to an increase of the cathodic current (due to the electrocatalytic reduction of the enzymatically produced hydrogen peroxide). The Paper Blue-based biosensor is capable to detect glucose linearly up to $25 \mathrm{mM}$ ( $450 \mathrm{mg} / \mathrm{dL})$ : it offers a valuable platform 
Wax printing
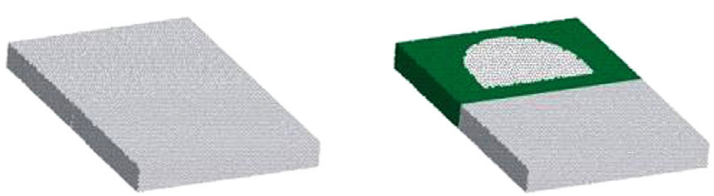

Screen-printing
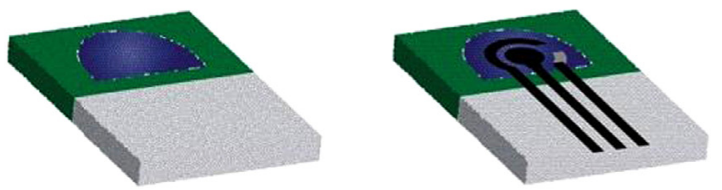
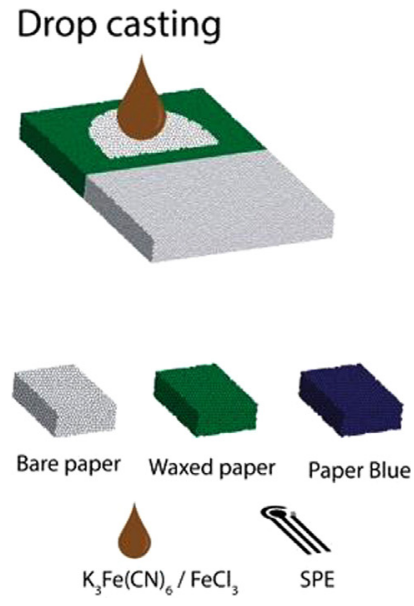

Fig. 3. Combination of Paper Blue with wax printing and screen-printing to produce electrochemical strips.
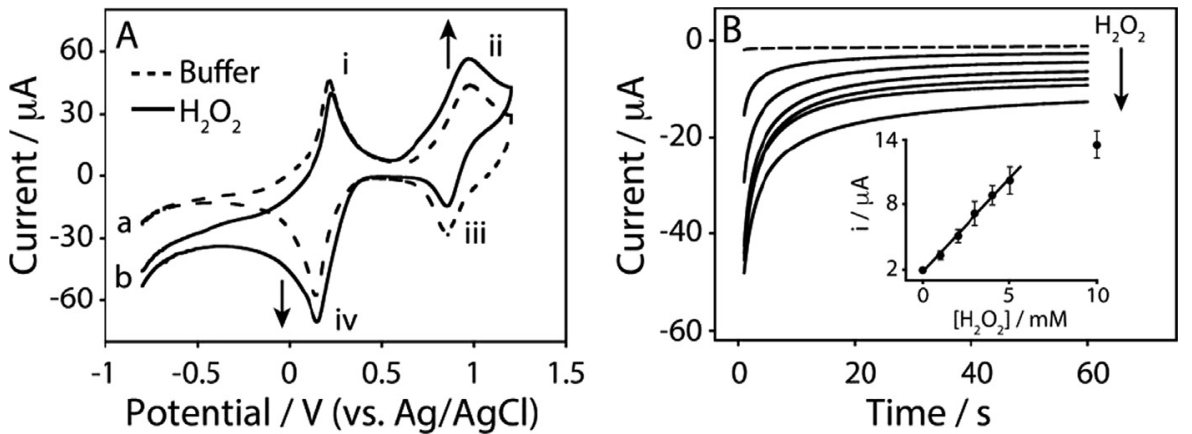

Fig. 4. A) Cyclic voltammetry experiments in (a) absence and in (b) presence of $5 \mathrm{mM}$ hydrogen peroxide at a scan rate of $0.02 \mathrm{~V} / \mathrm{s}$; B) Amperometric detection of increasing concentration of hydrogen peroxide (0-10 mM), applying $-0.1 \mathrm{~V}$. Inset: calibration curve of hydrogen peroxide in the range $0-10 \mathrm{mM}$. All the measurements have been carried out in $0.05 \mathrm{M}$ phosphate buffer ( $\mathrm{pH} 7.4)$. to monitor both normal and pathological conditions related to glucose level in blood. In addition, the selectivity of the Paper Blue-based biosensor has been evaluated in presence of common potential blood interferents, including ascorbic acid, uric acid, and acetaminophen ( $5 \mathrm{mM}$ for each interferent): the presence of these electroactive species has not showed significant effect on the response of the biosensor.

In this work many advantages should be taken into account in order to highlight the innovation related to the development of an entirely integrated paper-based sensing platform as in the case of Paper Blue. The extension of linearity and the possibility to measure in whole blood are key-features, and Paper Blue is amenable to meet with that. Many published platforms are limited within the 10-20 mM range [27-30] while the intrinsic porosity of Paper Blue confers the biosensor a wider application, avoiding the use of extra diffusion-limiting pads/membranes [31,32], or species that improve the $\mathrm{O}_{2}$ level for enzymatic reaction, i.e. perfluorocarbon $[33,34]$. Moreover, the possibility of realizing a device entirely integrated onto paper represents an ulterior improvement in respect to the state of the art. The use of paper-based substrates to detect glucose has been already reported through several published papers, i.e. detection of glucose by using a gold sputtered paper combined with an external screen-printed electrode [35], detection of glucose in beverages by using a paper-based platforms where
A
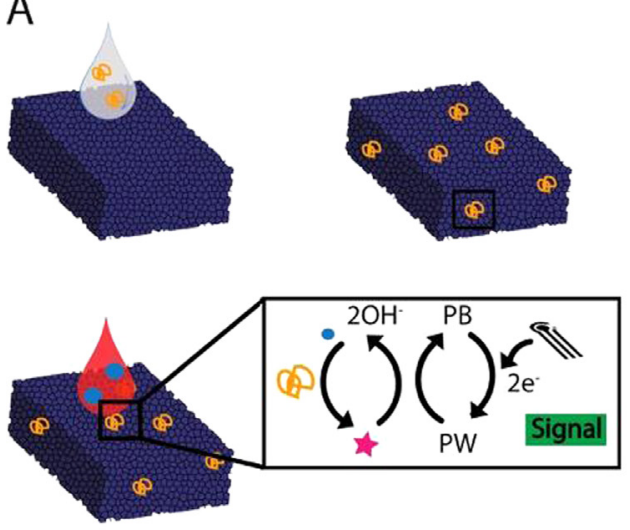

Pre-loaded reagents $\odot$ Glucose oxidase

Blood Glucose Hydrogen peroxide
B

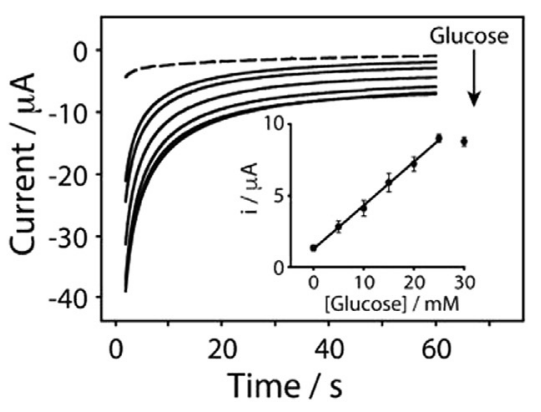

C

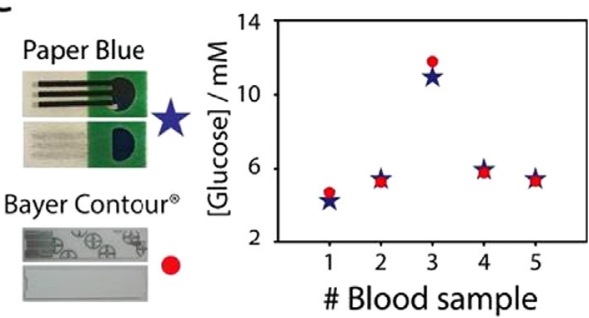

Fig. 5. A) Development and measurement scheme of the blood glucose biosensor on paper Blue; B) Amperometric detection of increasing concentration of glucose $(0-30 \mathrm{mM})$, applying $-0.1 \mathrm{~V}$. Inset: calibration curve of glucose in the range $0-30 \mathrm{mM}$ (measurements have been carried out in $0.05 \mathrm{M}$ phosphate buffer, pH 7.4); C) Paper Blue vs. Bayer Contour ${ }^{\circledR}$ for blood glucose determination. 
electrodes are also screen-printed [36], non-enzymatic detection by means of a cobalt phthalocyanine-ionic liquid-graphene composite drop casted onto the electrode [37]. Considering all the features that characterize these approaches, the platform developed in the present work simultaneously gathers three main aspects: the active role of cheap filter paper for synthesizing a smart material without expensive and/or time-consuming procedures, the realization of a fully-integrated device without the need of post-modification of the electrodes, and the application towards a complex biological matrix like the whole blood. To this regard, by measuring blood droplets provided by diabetic and nondiabetic volunteers, the performance of Paper Blue-based biosensor has been satisfactorily compared with commercially available glucose strips (Bayer Contour ${ }^{\circledast}$ ), obtaining a satisfactory correlation of 0.987 .

\section{Conclusions}

In conclusion, paper is amenable of several remarks:

i) It offers a micro-environment for the formation of nanoparticles, in a reagent-free approach;

ii) It is an eco-designed reactor: its porosity allows for the use of few $\mu \mathrm{L}$ of precursors, avoiding waste production;

iii) Paper Blue is easily combined with printed technologies to develop all-in-one strips for the use of non-specialists;

iv) The cellulosic network of Paper Blue is capable both filter a real matrix, i.e. whole blood, and to extend the linearity (usually obtained with extra-pad and/or oxygen-providing species);

v) It allows for the replacement of plastic-based commercial strips, used in glucose self-monitoring, with cheaper, more sustainable and easily disposable (incineration) substitutes.

This work opens to a plenty of different applications based on paper. It might serve as a reactor to create different nanomaterials, i.e. gold nanoparticles, as platforms for both electrochemical and colorimetric assays. Some of our preliminary results display interesting properties due to the synthesis features of paper in presence of gold precursors. Perhaps, future developments could be focused on the employment of papers with different characteristics, i.e. pores size, purification grade, possibly yielding materials with different (catalytic) properties. In addition to this insight, paper might serve as a remediation-active material, for instance by functionalizing it with a heavy metal-adsorbent material: one can imagine to remediate and detect the heavy metal with the same platform. Again, different functional paper sheets can be assembled together with the possibility to evolve towards 3D integrated multiplexed tools, going beyond the state of the art that purely highlights the use of paper as substrates where sensing device can be obtained.

\section{Acknowledgements}

S.C. acknowledges Fondazione Umberto Veronesi for "Postdoctoral Fellowship 2017 and 2018". Authors acknowledge Julian Ramirez for proofreading the manuscript, and Prof. Ilaria Cacciotti (University of Rome "Niccolò Cusano") for SEM experiments.

\section{References}

[1] H.M. Lee, S.Y. Choi, A. Jung, S.H. Ko, Highly conductive aluminum textile and paper for flexible and wearable electronics, Angew. Chem. Int. Ed. 52 (2013) $7718-7723$.

[2] M. Liu, C.Y. Hui, Q. Zhang, J. Gu, B. Kannan, S. Jahanshahi-Anbuhi, C.D.M. Filipe, J.D. Brennan, Y. Li, Target-induced and equipment-free DNA amplification with a simple paper device, Angew. Chem. 128 (2016) 27592763.

[3] M. d'Halluin, J. Rull-Barrull, G. Bretel, C. Labrugère, E. Le Grognec, F.X. Felpin, Chemically modified cellulose filter paper for heavy metal remediation in water, ACS Sustain. Chem. Eng. 5 (2017) 1965-1973.

[4] A.A. Kumar, J.W. Hennek, B.S. Smith, S. Kumar, P. Beattie, S. Jain, J.P. Rolland, T.P. Stossel, C. Chunda-Liyoka, G.M. Whitesides, From the bench to the field in low-cost diagnostics: two case studies, Angew. Chem. Int. Ed. 54 (2015) $5836-5853$.

[5] R. Pelton, Bioactive paper provides a low-cost platform for diagnostics, Trends Anal. Chem. 28 (2009) 925-942.

[6] A.K. Yetisen, M.S. Akram, C.R. Lowe, Paper-based microfluidic point-of-care diagnostic devices, Lab Chip 13 (2013) 2210-2251.

[7] S. Cinti, B. De Lellis, D. Moscone, F. Arduini, Sustainable monitoring of $\mathrm{Zn}[\mathrm{II}]$ in biological fluids using office paper, Sens. Actuators B 253 (2017) 1199-1206.

[8] N. Dempsey, G. Bramley, S. Power, C. Brown, The social dimension of sustainable development: defining urban social sustainability, Sustain. Dev. 19 (2011) 289-300.

[9] M. Li, J. Tian, M. Al-Tamimi, W. Shen, Paper-based blood typing device That reports patient's blood type "in writing", Angew. Chem. Int. Ed. 51 (2012) 5497-5501.

[10] P.T. Anastas, J.C. Warner, Green Chemistry: Theory and Practice, Oxford University Press, New York, 1998.

[11] F. Ricci, G. Palleschi, Sensor and biosensor preparation, optimisation and applications of Prussian Blue modified electrodes, Biosens. Bioelectron. 21 (2005) 389-407.

[12] M.A. Komkova, E.E. Karyakina, A.A. Karyakin, Noiseless performance of Prussian Blue based (bio)sensors through power generation, Anal. Chem. 89 (2017) 6290-6294.

[13] N.A. Sitnikova, M.A. Komkova, I.V. Khomyakova, E.E. Karyakina, A.A. Karyakin, Transition metal hexacyanoferrates in electrocatalysis of $\mathrm{H}_{2} \mathrm{O}_{2}$ reduction: an exclusive property of Prussian Blue, Anal. Chem. 86 (2014) 4131-4134.

[14] S. Cinti, F. Arduini, G. Vellucci, I. Cacciotti, F. Nanni, D. Moscone, Carbon black assisted tailoring of Prussian Blue nanoparticles to tune sensitivity and detection limit towards $\mathrm{H}_{2} \mathrm{O}_{2}$ by using screen-printed electrode, Electrochem. Commun. 47 (2014) 63-66.

[15] S. Cinti, C. Minotti, D. Moscone, G. Palleschi, F. Arduini, Fully integrated ready-touse paper-based electrochemical biosensor to detect nerve agents, Biosens. Bioelectron. 93 (2017) 46-51.

[16] S. Cinti, L. Fiore, R. Massoud, C. Cortese, D. Moscone, G. Palleschi, F. Arduini, Lowcost and reagent-free paper-based device to detect chloride ions in serum and sweat, Talanta 179 (2018) 186-192.

[17] A. Valiūnienè, A.I. Rekertaitè, A. Ramanavičienè, L. Mikoliūnaitė, A. Ramanavičius, Fast Fourier transformation electrochemical impedance spectroscopy for the investigation of inactivation of glucose biosensor based on graphite electrode modified by Prussian Blue, polypyrrole and glucose oxidase, Colloids Surf. A 532 (2017) 165-171.

[18] A. Valiūnienè, P. Virbickas, A.I. Rekertaitė, A. Ramanavičius, Amperometric glucose biosensor based on titanium electrode modified with Prussian Blue layer and immobilized glucose oxidase, J. Electrochem. Soc. 164 (2017) B781-B784.

[19] S. Cinti, M. Basso, D. Moscone, F. Arduini, A paper-based nanomodified electrochemical biosensor for ethanol detection in beers, Anal. Chim. Acta 960 (2017) $123-130$

[20] S. Cinti, F. Arduini, D. Moscone, G. Palleschi, L. Gonzalez-Macia, A.J. Killard, Cholesterol biosensor based on inkjet-printed Prussian Blue nanoparticle-modified screen-printed electrodes, Sens. Actuators B 221 (2015) 187-190.

[21] S. Imani, A.J. Bandodkar, A.V. Mohan, R. Kumar, S. Yu, J. Wang, P.P. Mercier, A wearable chemical-electrophysiological hybrid biosensing system for real-time health and fitness monitoring, Nat. Commun. 7 (2016) 11650.

[22] D. Moscone, D. D'ottavi, D. Compagnone, G. Palleschi, A. Amine, Construction and analytical characterization of Prussian Blue-based carbon paste electrodes and their assembly as oxidase enzyme sensors, Anal. Chem. 73 (2001) 2529-2535.

[23] A.P. Turner, Biosensors: sense and sensibility, Chem. Soc. Rev. 42 (2013) 3184-3196.

[24] E. Witkowska Nery, M. Kundys, P.S. Jelen, M. Jönsson-Niedziółka, Electrochemical glucose sensing: is there still room for improvement? Anal. Chem. 88 (2016) 11271-11282.

[25] E. Carrilho, A.W. Martinez, G.M. Whitesides, Understanding wax printing: a simple micropatterning process for paper-based microfluidics, Anal. Chem. 81 (2009) 7091-7095.

[26] C.W. Foster, R.O. Kadara, C.E. Banks, Fundamentals of Screen-printing Electrochemical Architectures, Screen-printing Electro-chemical Architectures, Springer International Publishing, Cham, 2016, pp. 13-23.

[27] J. Zhang, X. Yu, W. Guo, J. Qiu, X. Mou, A. Li, H. Liu, Construction of titanium dioxide nanorod/graphite microfiber hybrid electrodes for a high performance electrochemical glucose biosensor, Nanoscale 8 (2016) 9382-9389.

[28] A. Ramanavicius, A.I. Rekertaitè, R. Valiūnas, A. Valiūnienė, Single-step procedure for the modification of graphite electrode by composite layer based on polypyrrole, Prussian Blue and glucose oxidase, Sens. Actuators B 240 (2017) 220-223.

[29] W. Huang, S. Ding, Y. Chen, W. Hao, X. Lai, J. Peng, J. Tu, Y. Cao, X. Li, 3D NiO hollow sphere/reduced graphene oxide composite for high-performance glucose biosensor, Sci. Rep. 7 (2017) 5220

[30] O. Parlak, A. İncel, L. Uzun, A.P. Turner, A. Tiwari, Structuring Au nanoparticles on two-dimensional $\mathrm{MoS}_{2}$ nanosheets for electrochemical glucose biosensors, Biosens. Bioelectron. 89 (2017) 545-550.

[31] S. Myler, S.D. Collyer, K.A. Bridge, S.P.J. Higson, Ultra-thin-polysiloxane-filmcomposite membranes for the optimisation of amperometric oxidase enzyme electrodes, Biosens. Bioelectron. 17 (2002) 35-43.

[32] M. Scampicchio, A. Arecchi, N.S. Lawrence, S. Mannino, Nylon nanofibrous membrane for mediated glucose biosensing, Sens. Actuators B 145 (2010) 394-397.

[33] T. Matsumoto, A. Ohashi, N. Ito, H. Fujiwara, T.A. Matsumoto, Long-term lifetime amperometric glucose sensor with a perfluorocarbon polymer coating, Biosens. Bioelectron. 16 (2001) 271-276.

[34] M. Karaskiewicz, J.F. Biernat, J. Rogalski, K.P. Roberts, R. Bilewicz, Fluoroaromatic 
substituents attached to carbon nanotubes help to increase oxygen concentration on biocathode in biosensors and biofuel cells, Electrochim. Acta 112 (2013) 403-413.

[35] E. Núnez-Bajo, M.C. Blanco-López, A. Costa-García, M.T. Fernández-Abedul, In situ gold nanoparticle electrogeneration on gold films deposited on paper for non-enzymatic electrochemical determination of glucose, Talanta 178 (2018) 160-165.

[36] P.J. Lamas-Ardisana, G. Martínez-Paredes, L. Añorga, H.J. Grande, Glucose biosensor based on disposable electrochemical paper-based transducers fully fabricated by screen-printing, Biosens. Bioelectron. 109 (2018) 8-12.

[37] S. Chaiyo, E. Mehmeti, W. Siangproh, T.L. Hoang, H.P. Nguyen, O. Chailapakul, K. Kalcher, Non-enzymatic electrochemical detection of glucose with a disposable paper-based sensor using a cobalt phthalocyanine-ionic liquid-graphene composite, Biosens. Bioelectron. 102 (2018) 113-120. 